\title{
BMJ Open Prospective exploration of the effect of adiposity and associated microbial factors on healing and progression of diabetic foot ulcers in Tanzania: study protocol of a longitudinal cohort study
}

\author{
Fredirick Mashili (1) , ${ }^{1}$ Agricola Joachim, ${ }^{2}$ Said Aboud, ${ }^{2}$ Mabulla Mchembe, ${ }^{3}$ \\ Faraja Chiwanga, ${ }^{4}$ Juliet Addo, ${ }^{5}$ Lindsay Kendall, ${ }^{5}$ Agbor Ako, ${ }^{5}$ Zulfiqar Abbas ${ }^{6,7}$
}

To cite: Mashili F, Joachim A, Aboud S, et al. Prospective exploration of the effect of adiposity and associated microbial factors on healing and progression of diabetic foot ulcers in Tanzania: study protocol of a longitudinal cohort study. BMJ Open 2019;9:e031896. doi:10.1136/ bmjopen-2019-031896

- Prepublication history and additional material for this paper are available online. To view these files, please visit the journal online (http://dx.doi. org/10.1136/bmjopen-2019031896).

Received 24 May 2019 Revised 06 November 2019 Accepted 11 November 2019

Check for updates

(C) Author(s) (or their employer(s)) 2019. Re-use permitted under CC BY-NC. No commercial re-use. See rights and permissions. Published by BMJ.

For numbered affiliations see end of article.

Correspondence to Dr Fredirick Mashili; fredirick@gmail.com

\section{ABSTRACT}

Background Diabetic foot ulcers (DFUs) are associated with high morbidity and mortality in low-income countries. This coexists with an increasing prevalence of obesity which has been reported to alter antimicrobial susceptibility and potentially affect the outcome of infected foot ulcers. This study aims to determine whether adiposity and local microbial factors affect the progression and healing of foot ulcers in people with type 2 diabetes in hospital settings in Tanzania.

Methods and analysis A prospective cohort of 300 individuals with type 2 diabetes presenting with DFUs at an outpatient clinic will be enrolled into the study. At baseline, participants will be stratified into normal and high adiposity groups (150 per group) as measured by bioelectrical impedance analysis (BIA). Both groups will receive DFU management according to locally appropriate standards of care and will be followed up for 24 weeks or until complete wound healing, whichever occurs first. The primary end point is complete wound healing at 24 weeks while secondary end points are ulcer progression (worsening or improving), amputation and death. Enrolling 150 participants per group will have a minimum power of $80 \%$ to detect a $20 \%$ difference in cumulative incidence of complete ulcer healing (at the $5 \%$ level of statistical significance) between the normal and high adiposity groups.

Ethical considerations and dissemination of results This study will be conducted in compliance with the independent institutional review boards (IRBs), informed consent guidelines, the declaration of Helsinki and International Conference on Harmonisation, Good Clinical Practice Guidelines. Ethical clearance has been granted by the Muhimbili University of Health and Allied Sciences ethical review board (MUHAS Ref. No. DA.282/298/01 .C/). Permissions to conduct the study have been granted by the Abbas Medical Centre and the Muhimbili Academic Medical Centre (MAMC).

Progress and results emanating from this work will be communicated to the scientific community through conference presentations, short communications (using journal letters and interesting case reports) and peerreviewed publications. When necessary, through proper

\section{Strengths and limitations of this study}

- To our knowledge, this will be the first longitudinal study investigating the effect of body fat (adiposity) on the outcome (healing, amputation and death) of diabetic foot ulcers (DFUs) in a black African population.

- The prospective nature of the study will ensure standardisation of care hence minimising potential bias related to differences in standard of care between the two groups under comparison.

- The study will systematically evaluate adiposity using robust tools (bioelectrical impedance analysis technology and automated device to measure peripheral arterial insufficiency).

- The use of automated equipment will minimise interoperator/intraoperator variability and enhance standardisation and reproducibility.

- The main limitation is that while using predominantly one DFU care and treatment centre helps reduce potential bias from differing standards of care, the results will need to be validated in future studies using other centres before the findings could be generalised.

channels, popular means of communication (newspapers, magazines and online communications) will be used to inform policy and the public.

Trial registration number NCT03960255; Pre-results.

\section{INTRODUCTION}

Diabetes mellitus (DM) causes significant morbidity and mortality in sub-Saharan Africa (SSA). ${ }^{1-3}$ Among people with DM, diabetic foot ulcers (DFUs) constitute the largest proportion of admissions, amputations and mortality. ${ }^{2}{ }^{4-8}$ The prevalence of DFU in SSA ranges from $9.5 \%$ to $18.1 \% .^{19-11}$ Despite similar prevalence with that observed in other parts of the world, management of DFUs in SSA is challenging. As a result, DFUs 
contribute to higher rates of morbidity and mortality in people with DM. ${ }^{1}$ For example, in a Tanzanian study, among patients admitted with DFUs, 33\% had amputations. ${ }^{1}$ In addition, $54 \%$ of those with advanced pathology (Wagner Score $\geq 4$ ), died within 1 year. This is a relatively higher rate compared with what has been reported in the Western world. ${ }^{112}$

A growing body of evidence shows that DFUs in lowincome settings like SSA, often present with uncontrolled infections. ${ }^{2313}$ Due to limited accessibility to culture and sensitivity tests, these cases are usually treated empirically. However, empirical use of antibiotics in DFUs does not always guarantee successful infection control. A recent study from a Peruvian national hospital reported high rates of antibacterial resistance among patients with chronic DFUs. ${ }^{14}$ This resistance was observed for most of the antibiotics commonly used for empirical treatment of infection in DFUs. ${ }^{14}$ In connection with antimicrobial susceptibility patterns, studies are increasingly showing a link between obesity and complicated infection, as well as impaired susceptibility to antimicrobial agents. ${ }^{15-17}$ This could be due to significant changes in body composition (increasing body fat (BF)/adiposity), ${ }^{18}$ which characterise obesity, and may explain the observed antibiotic resistance related to DFUs.

Available evidence suggests that there may be a link between increased body mass index (BMI) (a surrogate measure of adiposity) and chronic DFUs. ${ }^{19-22}$ Since adiposity drives several functional impairments that are related to obesity and type 2 diabetes mellitus (T2DM), ${ }^{23}$ high adiposity may alter biological processes that are important in wound healing. For example, increased adipose mass is associated with decreased vascularity and impaired circulation which may compromise ulcer healing process. ${ }^{24-30}$ Impaired circulation/perfusion also impairs oxygen delivery, ${ }^{31-33}$ thereby encouraging the growth of anaerobic microbes as well as fungi. ${ }^{34}{ }^{35}$ Presence of anaerobic cocci can delay wound healing, ${ }^{35}$ partly explaining wound chronicity. These observations indicate that there may be a combined influence of adiposity and poor perfusion (impaired vascularisation, microvascular dysfunction) on the type and characteristics of microbial agents infecting DFUs.

Understanding local microbial flora may guide a more targeted use of antibiotics thereby minimising the potential for the emergence of antibiotic resistance. ${ }^{36} \mathrm{In}$ addition, studies conducted both in humans and animal models of diabetes show that a decrease in lean body mass (LBM) with or without an increase in adiposity can significantly delay wound healing due to insufficient structural and immune system proteins/cells such as collagen and fibroblasts. ${ }^{37-41}$ Indeed in patients with T2DM with advanced foot ulcers, a high protein diet resulted in a significant recreation of LBM and substantial improvement in DFUs. ${ }^{31} 3642$ Several other studies have demonstrated comparable improvements in wound healing with restoration of normal body composition. ${ }^{43-46}$ In most of those studies however, the main focus had been LBM and not adiposity. Given the deleterious biological effects that are related to high adiposity, this study seeks to elucidate the relationship between adiposity and delayed wound healing in DFUs.

\section{Objectives}

The overall primary objective is to determine the effect of adiposity and associated microbial factors on healing and progression of DFUs in people with T2DM. The specific objectives are to:

1. Determine the proportion of complete ulcer healing at 24 weeks, in patients with T2DM with normal and high adiposity.

2. Determine the proportion of improving or worsening DFUs at 24 weeks, in patients with T2DM with normal and high adiposity.

3. Determine whether there is an association between baseline adiposity (normal and high) and ulcer healing (complete healing at weeks, and/or healing time).

4. Determine whether baseline adiposity and microbial characteristics have dependent and/or combined associations with ulcer healing at 24 weeks.

5. Evaluate whether there is an association between adiposity and adverse outcomes in people with DFUs (amputation and death).

The objectives are based on our hypothesis that in people with DFUs high adiposity (dependently or independently) delays wound healing compared with normal adiposity (figure 1 ).

In addition, this project has secondary objectives (summarised in box 1)

\section{METHODS AND ANALYSIS}

\section{Study design and setting}

This is a prospective cohort study that will take place in clinical care settings of patients attending routine management of DFUs in Dar es salaam, Tanzania. Patients will be recruited from the Abbas Medical Centre (AMC), a specialised clinic that focuses on treatment and care of DFUs. This clinic which is in close proximity to the University, offers standardised management of DFUs. Using a single centre at this point will help to ensure uniformity in patients' foot care and potentially minimise bias. However, to ensure that the targeted sample size is obtained within the allocated timeline, the diabetes clinic at the university teaching hospital (Muhimbili University of Health and Allied Sciences (MUHAS) Academic Medical Centre) will be used as a backup recruitment centre.

\section{Recruitment procedures and sampling techniques}

Based on previous studies reporting the demographic characteristics of the region, ${ }^{3}$ patients aged 30 years and above will be recruited. All eligible participants will be enrolled until the required numbers are obtained for both the normal and high adiposity groups. A total of 150 participants with high adiposity will be recruited 


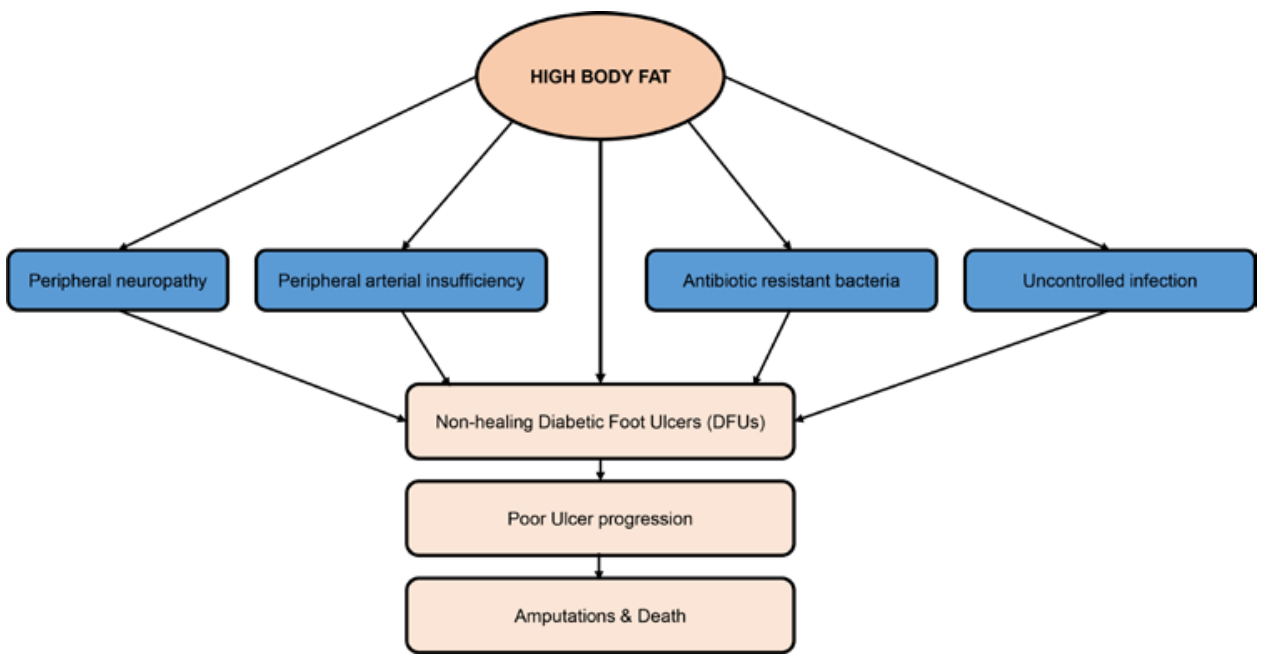

Figure 1 Conceptual framework diagram portraying possible connections between high body fat (high adiposity) and impaired healing of DFUs.

and matched, by both age ( \pm 5 years) and sex, to 150 participants with normal adiposity. The two groups will be followed up for a period of 24 weeks. Clinicians who usually attend to these patients will identify eligible participants, based on inclusion and exclusion criteria (box 2). Prior training on eligibility criteria and standard operating procedures will be provided to all clinicians and technicians involved in the study. Recruiting clinicians will enter information in electronic clinical report forms (linked to REDCap software) and will not be involved in data processing and analysis. Except for some assessment methods, all other DFU treatment and care will continue as usually practised at the clinic. All the procedures including patient recruitments will adhere to the principles of good clinical practice and will be performed by suitably qualified personnel. Routine practice at AMC

\section{Box 1 Secondary objectives}

To evaluate the validity of various baseline surrogate markers and measures of obesity/adiposity (body mass index (BMI), waist circumference, lean body mass (LBM) and phase angle) in predicting wound prognosis (healing, worsening, amputation or death) in people with diabetic foot ulcers (DFUs).

- To evaluate the validity of baseline measures of peripheral arterial function (peripheral pulses, ankle-brachial index (ABI), toe-brachial index (TBI), toe pressure and Doppler waveforms) in predicting wound prognosis (healing, worsening, amputation or death) in people with DFUs.

- To determine whether microbial characteristics (type, load, dominance/diversity and their antibiotic susceptibility patterns) differ in different stages/classifications of DFUs.

- To evaluate whether there is an association between microbial characteristics (type, load, dominance/diversity and their antibiotic susceptibility patterns), body composition, arterial status and glycaemic control.

- To evaluate whether baseline microbial characteristics (type, dominance, diversity and antibiotic susceptibility patterns) predict wound prognosis (healing, worsening, amputation or death) in people with DFUs. involves a number of steps that include a comprehensive assessment of various factors related to DFUs (history, physical examination and investigations). A specialist physician usually sees all the patients. Management of patients typically includes tight blood glucose control, wound care and standard double regimen antibiotics. This is usually guided by standardised protocols (mostly from the international working group for the diabetic foot).

\section{Case definition, risk groups and inclusion/exclusion criteria}

Participants will be newly reporting and known patients with T2DM with DFUs of any class, stage and grade (based on the university of Texas (UT) ulcer staging system). Any prior treatment and important information related to the ulcers and patients will be recorded using a pretested questionnaire. DFU will be defined as a full-thickness wound,

\section{Box 2 Inclusion and exclusion criteria}

\section{Inclusion criteria:}

- History of type 2 diabetes mellitus (T2DM).

- Presence of participant's signed informed consent.

- Age 30 years and above at the time the participant is signing the consent.

- Tanzanians of African origin (black Tanzanians).

- Presence of DFUs (new or recurrent) as defined in the method section of the manuscript.

\section{Exclusion criteria}

- Known patients with congestive cardiac and/or renal failure.

- Any patients with absolute or relative contraindication for tissue biopsy (for incident cases).

- Patients with obvious signs of advanced gangrene (dark discolouration of skin on a specific part of a limb accompanied by severe ischaemia).

- Patients with DFU with a non-healing ulcer of more than 52 weeks duration.

- Patients with DFU with below normal body mass index (BMI below 18). 


\section{Box 3 Secondary outcomes measures}

Secondary outcome measures (intermediate within 2 years of initial study)

- Time to complete healing, that is, speed of healing.

- Rate of ulcer recurrence (after healing) at 24 weeks.

- Improvement to a less severe or worsening to a more advanced ulcer stage over a period of 24 weeks (based on University of Texas ulcer classification).

- Rate of wound closure (percentage reduction in wound surface area over time).

- Resolution/appearance of infection based on clinical judgement.

Secondary outcome measure (long-term follow-up as part of scaling up)

- Amputation rates.

Mortality rates.

through the dermis, below the ankle in an individual with T2DM. Duration of ulcers will be measured and reported in weeks. Patients with non-healing ulcer of more than 52 weeks duration will be excluded (see box 3 for inclusion and exclusion criteria). The Infectious Diseases Society of America criteria will be used to define DFU infection. ${ }^{47}$ To recruit appropriate participants, a careful examination will be done with qualified and trained personnel. Prior to commencement of the study, appropriate manuals and standard operating procedures will be prepared and used throughout the study period.

\section{Exposure/risk groups and matching}

Primary exposure/risk factor investigated here is increased/high adiposity. At baseline, participants (patients with T2DM with DFUs) will be categorised into two groups based on percentage $\mathrm{BF}(\% \mathrm{BF})$ namely: normal and high adiposity groups (figure 2). The American Council on Exercise's obesity definition by $\% \mathrm{BF}$ will be used to categorise patients $(\% \mathrm{BF} \geq 25 \%$ and $\geq 32 \%$ in men and women respectively, will be categorised as high adiposity). ${ }^{48}$ The ratio of overweight and obesity to normal weight (based on BMI) among patients attending the AMC is around 2:1. But since the criteria to group the patients is based on \% BF (adiposity) as measured by bioelectrical impedance analysis (BIA) technology, we will apply a 1:1 recruitment protocol, whereby equal numbers of patients with normal and raised $\mathrm{BF}$ will be recruited. High and normal adiposity groups will also be matched for age ( \pm 5 years) and sex. Equal numbers of subjects with normal and impaired adiposity will be recruited for each sex.

\section{Outcome measures}

The primary end point (outcome measure) for this study is the proportion of individuals with complete ulcer/wound

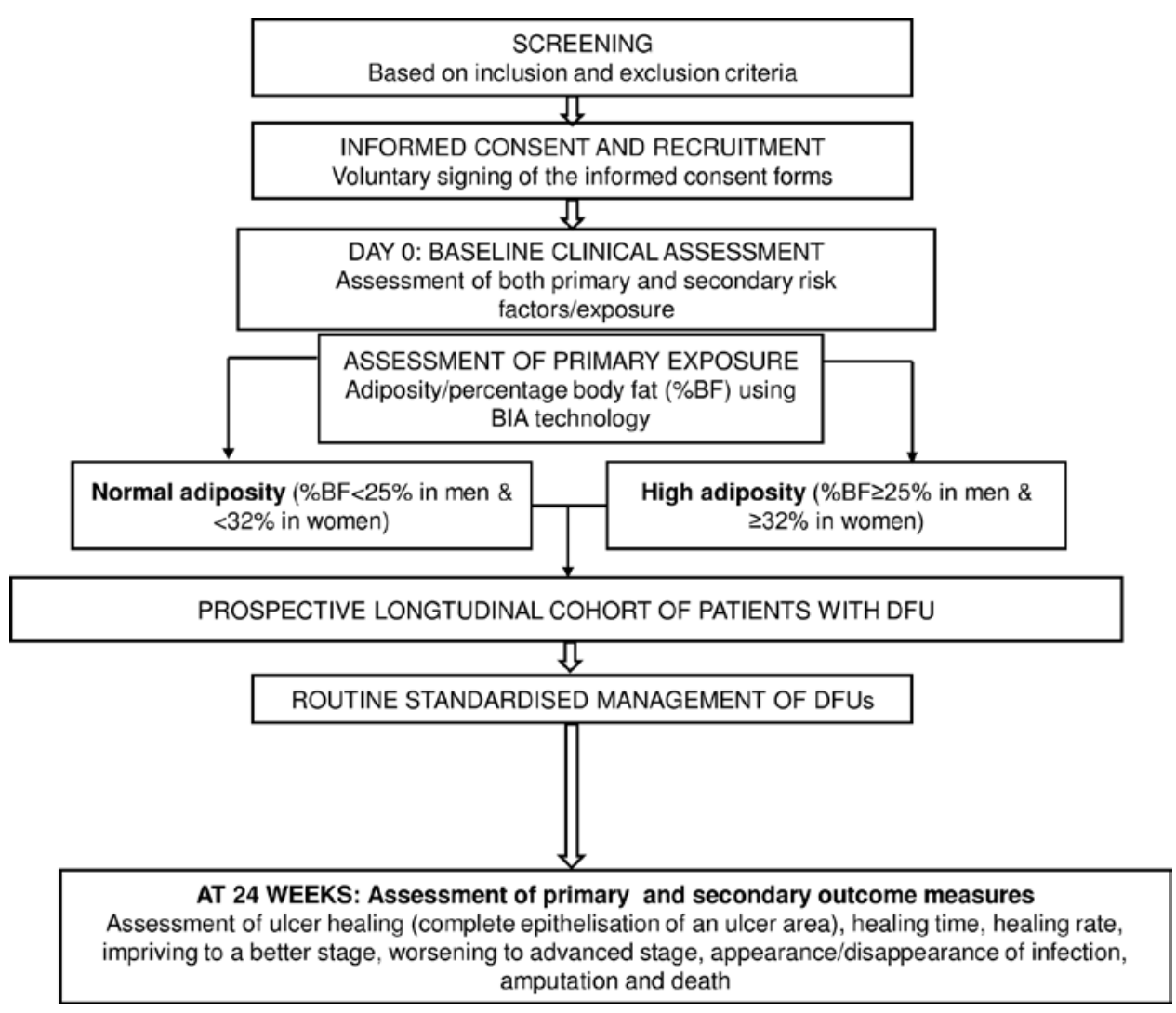

Figure 2 Study design summary showing the exposure/risk factors of interest and outcome measures. The American Council on Exercise's obesity definition by \%BF, will be used to categorise patients (\%BF $\geq 25 \%$ and $\geq 32 \%$ in men and women, respectively, will be categorised as high adiposity). BIA, bioelectrical impedance analysis; \%BF, percentage body fat; DFU, diabetic foot ulcer. 
healing at 24 weeks. Complete healing will be defined based on the criteria of the US Food and Drug Authority, as $100 \%$ re-epithelialisation of the wound surface (complete wound closure) with a complete absence of drainage and no requirement of dressing confirmed at two consecutive study visits, 2 weeks apart. ${ }^{49}$ The long follow-up period proposed in this study (24 weeks instead of the usual 12 weeks) was decided to accommodate 1 month posthealing confirmation. Photos of the ulcers will also be taken during follow-up. The proportion of patients with complete ulcer healing at 24 weeks among the group with impaired adiposity (increased \% BF) will be compared with that of controls (normal adiposity/ normal \% BF) using appropriate statistical approaches. In addition, the study will also have secondary outcome measures that are outlined in box 3 .

\section{Clinical procedures/measurements}

After being enrolled in the study based on inclusion and exclusion criteria, patients will be scheduled for assessment and data collection. On this day, all the clinical assessments and sample collection will be done. Questionnaires will also be filled on the same day.

The following tools and approaches will be used to collect information from the study participants:

1. Questionnaire and physical examination: A structured and pretested questionnaire will be incorporated into the REDCap software and will be used to collect data (online supplemental appendix 01). The questionnaire will be tested by administering it to a selected group of patients to see whether the questions capture what is intended. This will be done before the tool/questionnaire is incorporated into the REDCap software. An end of study (follow-up questionnaire) will also be used (online supplemental appendix 02). Having been incorporated the software will also be tested to make sure it functions properly.

The questionnaires will be supplemented with thorough case notes review and physical examination. Key information to be gathered will include baseline demographics; time since diagnosis of diabetes; ulcer characteristics (wound duration and location, aetiology, Perfusion, Depth, Extent, Infection and Sensation (PEDIS) and UT classification); antimicrobial treatment history including type of antimicrobial used; and if available any microbiology results from swab and tissue samples taken from the ulcer. Study clinicians will assess the patients for deformity (claw toes, prominent metatarsal heads hallux valgus and others). Relevant general and specific system examination will be done as usual, with focus on signs of infection, cardiovascular system abnormalities, peripheral neuropathy and vasculopathy.

2. Anthropometry: Weight and height will be measured in all subjects using standard protocols. ${ }^{50} \mathrm{BMI}$ will be calculated using the formula BMI=weight $(\mathrm{kg}) /$ height $\left(\mathrm{m}^{2}\right)$. Waist circumference will also be measured using a standard and pretested protocol. ${ }^{50}$ This will provide important data as covariates that will be used in statistical adjustment.

3. Body composition measurements: BIA, using a multifrequency Quadscan 4000 analyser (Bodystat, Isle of Man, UK), will be used to measure body composition (LBM, $\% \mathrm{BF}$ and phase angle). This involves applying a very small electrical current into the body to measure differences in electrical conductivity of BF and LBM. ${ }^{51}$ Based on the American Council on Exercise's obesity definition, $\% \mathrm{BF} \geq 25 \%$ and $\geq 32 \%$ in men and women, respectively, will be categorised as high adiposity while $\% \mathrm{BF}<25 \%$ and $<32 \%$ in men and women, respectively, will be categorised as normal adiposity. ${ }^{48}$ The measurements will follow standard protocols for whole body and segmental assessments, and will use appropriate BIA equations. ${ }^{52}$

4. Ulcer staging and classification: Ulcers will be classified as either neuropathic, ischaemic or neuroischaemic, based on the presence or absence of neuropathy or peripheral arterial disease (PAD). In addition, staging will be done based on the UT ulcer staging system. ${ }^{53}$ The UT classification was selected since ischaemia and infection are part of the grading system and will be thoroughly evaluated in the proposed study.

5. Assessment of peripheral Arterial status: Spectral Doppler (Duplex) sonography is well accepted as a non-invasive imaging modality to be used as a diagnostic test for detecting and grading the presence and severity of arterial disease. ${ }^{54} \mathrm{~A}$ relatively simpler physiological assessment using pulse volume recording (PVR) that record pulse volume waveforms (PVWs) has a strong correlation with spectral Doppler waveforms,${ }^{55}$ and will be used to identify PAD. Waveforms will be interpreted and a four-level pulse wave grading system will be used to define PAD. ${ }^{56} 57$

Presence or absence of significant ischaemia (or presence or absence of PAD) will also be assessed with the measurement of ankle-brachial index (ABI), as well as toe-brachial index (TBI). These involve the measurement of peak systolic flow velocities in the ankles (dorsalis pedis and posterior tibia arteries), toes and arms (brachial artery) by using an automated oscillometric device that measures ABI, TBI and PVW (Smartdop XT, Kodymedics, India). Toe blood pressure will simultaneously be measured. The use of automated devices will simplify the test and help to reduce operator-related errors and variability. An $\mathrm{ABI}$ of $>0.9-1.3$ will indicate normal flow, 0.6-0.9 mild ischaemia, 0.4-0.6 moderate ischaemia and $<0.4$ severe ischaemia. ${ }^{58}$ For simplicity and analysis an ABI of $>0.9$ to 1.3 will be considered as normal (no PAD) and $\leq 0.9$ as abnormal (presence of PAD). A TBI of $\geq 0.7$ will be considered normal, while TBI of $<0.7$ will be considered abnormal. PAD will be considered when any of the two measurements fall within the abnormal category.

6. Assessment of peripheral neuropathy: Peripheral neuropathy will be assessed based on previously published protocols, and appropriate scoring systems will be used for 
classification. ${ }^{59-62}$ Diabetic Neuropathy Examination Score ${ }^{61} 10 \mathrm{~g}$ Semmes-Weinstein monofilament examination and Quantitative Sensory Testing by Vibration Perception Threshold ${ }^{62}$ will be used to describe peripheral nerve function and define peripheral neuropathy. Diagnosis of neuropathy will be made when any of the scoring systems appear positive. Based on the presence or absence of pain, neuropathy will further be classified into painful and painless neuropathy, respectively.

\section{Laboratory procedures}

On the day of data collection qualified and trained personnel will draw fasting venous blood from all the subjects. This blood will be processed ready for storage. Blood storage and analysis will take place at the MUHAS microbiology laboratory.

1. Blood chemistry: A variety of biochemical parameters will be measured in blood using standardised methods and protocols. This will include lipid profiles (total cholesterol, low density lipoprotein cholesterol (LDL), high density lipoprotein cholesterol (HDL), triglyceride (TG)) and glycosylated haemoglobin. Fibroblast growth factor 21, a promising adiposity maker will also be measured in serum at baseline and at 24 weeks. Most of these biochemical parameters will be compared between the two groups (normal and increased adiposity groups) at baseline and their utility in predicting ulcer progression/healing will be evaluated.

2. Specimen collection, identification and susceptibility testing: After cleaning the wound/ulcer with sterile saline, tissue biopsy will be taken for culture using a special punch biopsy device. Biopsies will be taken in all patients reporting at the clinic with DFU for the first time (incident cases). This is important to avoid the effect of prior antibiotics and other treatments on the ulcer microbiology. In case of necrotic wounds, debridement will be done under local anaesthesia (2\% lignocaine) before taking the biopsy. Specimens obtained will be handled appropriately before being transported to the laboratory and processed within an hour of collection as per standard operating procedures. Finally, the specimen will be cultured in selective and non-selective agar media under anaerobic and aerobic conditions.

Inoculated culture plates will be incubated aerobically at $37^{\circ} \mathrm{C}$ and anaerobically using the conventional Gas-pack system (Oxford, UK). Inhouse biochemical tests will be used to identify bacterial and fungal growth. Susceptibility of the isolates to various antimicrobials will be performed using the disc diffusion method as recommended by the Clinical Laboratory Standards Institute guidelines. ${ }^{63}{ }^{64}$ Uncultured bacteria which are common in DFUs cannot be identified by the diagnostic methods employed in this study. It is acknowledged that this study might underestimate the pathogens present in DFUs.

Bacteria will be characterised based on their type/strain. Microbial load/density will be determined semiquantitatively (scant, light, moderate or heavy). Dominance and diversity will be defined as the predominance of one type of microorganism relative to others and the presence of multiple microorganisms, respectively. However, dominance and diversity indices can well be assessed by molecular techniques, a future consideration in project scaling up. Presence of microbial agents with signs and symptoms of infections will be classified as infection caused by the isolated microbes.

Tissue biopsy which is superior to the swab technique in detecting various microbes across all the ulcer grades ${ }^{65}$ was chosen for obtaining culture samples.

\section{Sample size calculations and analysis plan}

1. Sample size calculation: We estimated the required sample size based on power calculations. A $20 \%$ difference in DFU healing at 24 weeks, between the high and normal adiposity groups will be considered as a clinically meaningful difference. To attain a power of $80 \%$ at $95 \% \mathrm{CI}$ and aiming to recruit equal numbers of patients with DFU in the two groups (normal and high adiposity), a total sample size of 188 subjects is required. This calculation is based on the assumptions that the proportions (cumulative incidences) of complete wound healing at 24 weeks in normal and high adiposity groups are $55 \%$ and $35 \%$, respectively. This assumption was based on clinical records of patients with complete wound healing at 24 weeks, who were attending DFU clinics in Dar es Salaam (unpublished data). These patients were predominantly overweight and obese hence the proportion (35\%) has been used for the high adiposity group. Assuming a maximum of $20 \%$ loss to follow-up and considering the presence of competing risks (lower limb amputation and death) we plan to recruit a total of 300 patients with DFU (150 in each group) to maintain the power that might be weakened by a decrease in sample size due to loss to follow-up, and also to maintain the effect size that might be affected by competing risks (lower limb amputation and death).

2. Primary outcome analysis: The cumulative incidence competing risk method will be used. This method takes into account all the different events/outcomes that may affect the outcome of interest ${ }^{66}$ in this case, amputation and death.

At 24 weeks, we will summarise the outcome of categorised study participants (normal and raised adiposity groups) by using the proportions of complete wound healing in the two groups. These proportions will be estimated from the cumulative incidence (rate) of healing at 24 weeks (with death and amputation as competing risks) and compare the incidences (proportions) between normal and raised adiposity groups (using $\chi^{2}$ or Grey's test). ${ }^{67}$ In this regard healing estimates and cumulative incidence curves of the time to healing, in the presence of competing risks will be provided with 95\% CIs. Participants who will not experience any adverse event (amputation or death) before and at the end of initial follow-up (24 weeks) will be censored. 
To find out whether there is any significant difference in healing between patients with normal and those with high adiposity, we will use the subdistribution proportional hazards mode in a multivariate survival analysis to compare the two groups by examining the difference in the hazards of healing between normal and high adiposity groups (adjusting for known covariates including baseline ulcer characteristics that describe severity including ulcer surface area, stage, presence of infection and others). Similar analysis will be done to compare the outcome of grouped study participants based on ulcer-related events that are our secondary end points (outcome measures).

3. Descriptive analysis: Exploratory analysis beyond the primary study design will be done among the incident cases at baseline and different follow-up points.

Descriptive and inferential statistics will be used to determine the characteristics of DFUs in patients with T2DM. Measured variables will include type and load/density of microorganisms and their characteristics (dominance, diversity and antimicrobial sensitivity pattern). Related factors are systemic factors such as adiposity, circulatory insufficiency, impaired LBM, poor glycaemic control and behavioural characteristics like cigarette smoking, dietary habits and physical activity type/levels. Local factors including ulcer types/grades/classes will also be considered. Univariate analysis that includes measures of distribution, central tendency and dispersion will be used. Frequency of occurrences of different types and characteristics of microorganisms will be measured using proportions/means/median and compared using appropriate statistical tests, depending on the nature of the analysed data (t-test, analysis of variance and $\chi^{2}$ ).

Linear and logistic regression models will also be used to determine systemic factors that predict or explain local DFU characteristics for continuous and categorical data, respectively.

4. Predictive validity testing: Given its longitudinal nature, this study will offer an opportunity to evaluate the utility/ability of various assessment methods (physical examination, ABI, TBI, PVR and combined ABI and PVR) in predicting wound healing in an African population. We will calculate sensitivity and specificity of different methods (feeling peripheral pulses and ABI with PVWs) against ulcer outcomes (healed or not healed) at different follow-up points. Both independent and combined validity of $\mathrm{ABI}$ and PVW will be evaluated against ulcer outcomes. The receiver operating characteristics curve will be used to compare the diagnostic performance of the different assessment methods in predicting wound healing and also to set up reasonable cut-off points for the different tests.

Sensitivity, specificity, positive and negative predictive values, as well as accuracy will be calculated.

\section{Ethics and dissemination}

The aims, methods and all the study procedures including potential risks, will be explained to the participants before they consent to participate. Prior to any procedure, consenting participants will be asked to sign an informed consent form.

Results from this study may provide evidence for the role of adiposity and/or associated microbial factors on the outcome of DFUs in a Tanzanian clinical setting and may ultimately inform strategies to optimise the management of DFU and avert associated complications.

\section{Potential risks and mitigation plans}

Reported problems associated with tissue biopsy are rare but any invasive procedure has potential risks. To minimise risks, qualified and trained clinicians will perform the procedures under appropriate environmental conditions. Having been properly managed, adverse reactions or events (such as severe infection, falls, amputations and deaths) related to the study will be properly reported and documented.

\section{Time frames and milestones}

Recruitment will start in September 2019. The study is planned to end in March 2021.

\section{Patients and public involvement}

Patients will be involved in the designing, data collection and result dissemination. We have engaged patients in fine-tuning the questionnaire and other data collection tools during the study pilot period. During this period, patients have provided feedback on the questions, time spent and other aspects of data collection. Questionnaires were corrected based on feedback obtained from the patients. To further engage the patients, detailed explanation of all the procedures involved, and will be provided to all patients before and during data collection. In addition, all enrolled patients will be given their test results.

\section{Author affiliations}

${ }^{1}$ Physiology, Muhimbili University of Health and Allied Sciences, Dar es Salam, Tanzania

${ }^{2}$ Microbiology and Immunology, Muhimbili University of Health and Allied Sciences, Dar es Salaam, Tanzania

${ }^{3}$ Surgery, Muhimbili University of Health and Allied Sciences, Dar es Salaam, Tanzania

${ }^{4}$ Endocrinology and Diabetes, Muhimbili National Hospital, Dar es Salaam, Tanzania ${ }^{5}$ Global catalyst, GlaxoSmithKline Research and Development, Stevenage, UK ${ }^{6}$ Internal medicine, Muhimbili University of Health and Allied Sciences, Dar es Salaam, Tanzania

${ }^{7}$ Medicine, Abbas Medical Center, Dar es Salam, Tanzania

\section{Twitter Fredirick Mashili @fmashili}

Contributors FM conceptualised the study, led the development of the study protocol, and wrote the manuscript. AJ, SA, FC and MM contributed in the development of specific sections of the protocol and reviewed the manuscript. JA, LK, AA and ZA critically reviewed both the protocol and the manuscript. All authors provided valuable input and approved the final version of the manuscript.

Funding The study is funded by the GlaxoSmithKline R\&D (Africa NonCommunicable Disease Open lab grant), grant number 3000034576. The funder 
will not participate in data collection or any activity that is directly related to the execution of the research.

Competing interests JA, LK and AA are employees and shareholders of GlaxoSmithKline. They were only involved in the review and editing of the protocol and the manuscript. They will not be involved in data collection or any activity that is directly related to the execution of the research.

Patient consent for publication Not required.

Provenance and peer review Not commissioned; externally peer reviewed.

Open access This is an open access article distributed in accordance with the Creative Commons Attribution Non Commercial (CC BY-NC 4.0) license, which permits others to distribute, remix, adapt, build upon this work non-commercially, and license their derivative works on different terms, provided the original work is properly cited, appropriate credit is given, any changes made indicated, and the use is non-commercial. See: http://creativecommons.org/licenses/by-nc/4.0/.

ORCID iD

Fredirick Mashili http://orcid.org/0000-0002-5306-6703

\section{REFERENCES}

1 Gulam-Abbas Z, Lutale JK, Morbach S, et al. Clinical outcome of diabetes patients hospitalized with foot ulcers, Dar ES Salaam, Tanzania. Diabet Med 2002;19:575-9.

2 Abbas ZG, Archibald LK. Epidemiology of the diabetic foot in Africa. Med Sci Monit 2005;11:RA262-70.

3 Chalya PL, Mabula JB, Dass RM, et al. Surgical management of diabetic foot ulcers: a Tanzanian university teaching hospital experience. BMC Res Notes 2011;4:1-7.

4 Ndip EAA, Tchakonte B, Mbanya J-C, a A E, Ndip B. A study of the prevalence and risk factors of foot problems in a population of diabetic patients in Cameroon. Int $J$ Low Extrem Wounds 2006;5:83-8.

5 Kengne AP, Choukem SP, Deyahem YM, et al. Diabetic foot ulcers in Cameroon: can microflora prevalence inform probabilistic antibiotic treatment? J Wound Care 2006;15:363-6.

6 Tchakonté B, Ndip A, Aubry P, et al. [The diabetic foot in Cameroon]. Bull Soc Pathol Exot 2005;98:94-8.

7 Kidmas AT, Nwadiaro CH, Igun GO. Lower limb amputation in Jos, Nigeria. East Afr Med J 2004;81:427-9.

8 Abbas ZG, Gill GV, Archibald LK. The epidemiology of diabetic limb sepsis: an African perspective. Diabet Med 2002;19:895-9.

9 Kengne AP, Djouogo CFT, Dehayem MY, et al. Admission trends over 8 years for diabetic foot ulceration in a specialized diabetes unit in Cameroon. Int J Low Extrem Wounds 2009;8:180-6.

10 Ogbera AO, Fasanmade O, Ohwovoriole AE, et al. An assessment of the disease burden of foot ulcers in patients with diabetes mellitus attending a teaching hospital in Lagos, Nigeria. Int $J$ Low Extrem Wounds 2006;5:244-9.

11 Almobarak AO, Awadalla H, Osman M, et al. Prevalence of diabetic foot ulceration and associated risk factors: an old and still major public health problem in Khartoum, Sudan? Ann Trans/ Med 2017;5:340.

12 Ndosi M, Wright-Hughes A, Brown S, et al. Prognosis of the infected diabetic foot ulcer: a 12-month prospective observational study. Diabet Med 2018;35:78-88.

13 Morbach S, Lutale JK, Viswanathan V, et al. Regional differences in risk factors and clinical presentation of diabetic foot lesions. Diabet Med 2004;21:91-5.

14 Yovera-Aldana M, Rodríguez A, Vargas M, et al. Bacterial resistance in patients with infected diabetic foot without major amputation outcome in a Peruvian national Hospital, 2017.

15 Dhurandhar NV, Bailey D, Thomas D. Interaction of obesity and infections. Obes Rev 2015;16:1017-29.

16 Thjodleifsson B, Olafsson I, Gislason D, et al. Infections and obesity: a multinational epidemiological study. Scand J Infect Dis 2008;40:381-6.

17 Falagas ME, Kompoti M. Obesity and infection. Lancet Infect Dis 2006:6:438-46.

18 Dulloo AG, Jacquet J, Solinas G, et al. "Body composition phenotypes in pathways to obesity and the metabolic syndrome,". Int. J. Obes 2010.

19 Pinzur M, Freeland R, Juknelis D. The association between body mass index and foot disorders in diabetic patients. Foot Ankle Int. 2005;26:375-7.

20 Sohn M-W, Budiman-Mak E, Lee TA, et al. Significant J-shaped association between body mass index (BMI) and diabetic foot ulcers. Diabetes Metab Res Rev 2011;27:402-9.
21 Assaad-Khalil SH, Zaki A, Rehim AA, et al. Prevalence of diabetic foot disorders and related risk factors among Egyptian subjects with diabetes. Prim Care Diabetes 2015;9:297-303.

22 Tanamas SK, Wluka AE, Berry P, et al. Relationship between obesity and foot pain and its association with fat mass, fat distribution, and muscle mass. Arthritis Care Res 2012;64:262-8.

23 Algoblan A, Alalfi M, Khan M. Mechanism linking diabetes mellitus and obesity. DMSO 2014.

24 Pierpont YN, Dinh TP, Salas RE, et al. Obesity and surgical wound healing: a current review. ISRN Obes 2014;2014:1-13.

25 de Jongh RT, Serné EH, IJzerman RG, et al. Impaired microvascular function in obesity: implications for obesity-associated microangiopathy, hypertension, and insulin resistance. Circulation 2004;109:2529-35.

26 Schlager O, Willfort-Ehringer A, Hammer A, et al. Microvascular function is impaired in children with morbid obesity. Vasc Med 2011;16:97-102.

27 Jonk AM, Houben AJ, Schaper NC, et al. Obesity is associated with impaired endothelial function in the postprandial state. Microvasc Res 2011:82:423-9.

28 Farb MG, Ganley-Leal L, Mott M, et al. Arteriolar function in visceral adipose tissue is impaired in human obesity. Arterioscler Thromb Vasc Biol 2012;32:467-73.

29 Al-Tahami BAM, Ismail AAA-S, Bee YTG, et al. The effects of antiobesity intervention with orlistat and sibutramine on microvascular endothelial function. Clin Hemorheol Microcirc 2015;59:323-34.

30 Levy BI, Schiffrin EL, Mourad J-J, et al. Impaired tissue perfusion: a pathology common to hypertension, obesity, and diabetes mellitus. Circulation 2008;118:968-76.

31 Tatti P, di Mauro P, Neri M, et al. Reduced body cell mass in type 2 diabetes mellitus: reversal with a diabetes-specific nutritional formula. Med J Nutrition Metab 2010;3:133-6.

32 Koya D, Haneda M, Inomata S, et al. Long-Term effect of modification of dietary protein intake on the progression of diabetic nephropathy: a randomised controlled trial. Diabetologia 2009;52:2037-45.

33 Biolo G, Declan Fleming RY, Wolfe RR. Physiologic hyperinsulinemia stimulates protein synthesis and enhances transport of selected amino acids in human skeletal muscle. $J$ Clin Invest 1995;95:811-9.

34 Kalan L, Loesche M, Hodkinson BP, et al. Redefining the ChronicWound microbiome: fungal communities are prevalent, dynamic, and associated with delayed healing. MBio 2016;7:e01058-16.

35 Wall IB, Davies CE, Hill KE, et al. Potential role of anaerobic cocci in impaired human wound healing. Wound Repair Regen 2002;10:346-53.

36 Tatti P, Barber A. Nutritional Treatment of DiabeticFoot Ulcers - A Key to Success. In: Dinh T, ed. Global perspective on diabetic foot ulcerations, 2011.

37 Slavkovsky R, Kohlerova R, Tkacova V, et al. Zucker diabetic fatty rat: a new model of impaired cutaneous wound repair with type II diabetes mellitus and obesity. Wound Repair Regen 2011;19:515-25.

38 Pence BD, DiPietro LA, Woods JA. Exercise speeds cutaneous wound healing in high-fat diet-induced obese mice. Med Sci Sports Exerc 2012;44:1846-54.

39 Seitz O, Schürmann C, Hermes N, Frank S, et al. Wound healing in mice with high-fat diet- or ob Gene-induced diabetesobesity syndromes: a comparative study. Exp Diabetes Res 2010;2010:476969.

40 Delamaire M, Maugendre D, Moreno M, et al. Impaired leucocyte functions in diabetic patients. Diabet Med 1997;14:29-34.

41 Davidson NJ, Sowden JM, Fletcher J. Defective phagocytosis in insulin controlled diabetics: evidence for a reaction between glucose and opsonising proteins. J Clin Pathol 1984;37:783-6.

42 Tatti P, Barber A, di Mauro P, et al. Nutritional supplement is associated with a reduction in healing time and improvement of fat free body mass in patients with diabetic foot ulcers. Eur Wound Manag Assoc J 2010;10:13-18.

43 Moore MF, Dobson N, Castellino L, et al. Phase angle, an alternative physiological tool to assess wound treatment in chronic nonhealing wounds. J Am Col Certif Wound Spec 2011;3:2-7.

44 Fonder MA, Lazarus GS, Cowan DA, et al. Treating the chronic wound: a practical approach to the care of nonhealing wounds and wound care dressings. J Am Acad Dermatol 2008;58:185-206.

45 Frykberg RG, Driver VR, Carman D, et al. Chronic wounds treated with a physiologically relevant concentration of platelet-rich plasma gel: a prospective case series. Ostomy Wound Manage 2010;56:36-44.

46 Trengove NJ, Langton SR, Stacey MC. Biochemical analysis of wound fluid from nonhealing and healing chronic leg ulcers. Wound Repair Regen 1996;4:234-9. 
47 Lipsky BA, Peters EJG, Senneville E, et al. Expert opinion on the management of infections in the diabetic foot. Diabetes Metab Res Rev 2012;28 Suppl 1:163-78.

48 Waisbren E, Rosen H, Bader AM, et al. Percent body fat and prediction of surgical site infection. J Am Coll Surg 2010;210:381-9.

49 Snyder DL, Sullivan N, Schoelles KM. Skin Substitutes for Treating Chronic Wounds - Technology Assessment. Agency Healthc Res Qual 2012.

50 Centres for Disease Control and Prevention. Anthropometry procedures manual, 2007.

51 Faria SL, Faria OP, Cardeal MDA, et al. Validation study of multi-frequency bioelectrical impedance with dual-energy X-ray absorptiometry among obese patients. Obes Surg 2014;24:1476-80.

52 Ling CHY, de Craen AJM, Slagboom PE, et al. Accuracy of direct segmental multi-frequency bioimpedance analysis in the assessment of total body and segmental body composition in middle-aged adult population. Clin Nutr 2011;30:610-5.

53 Oyibo SO, Jude EB, Tarawneh I, et al. A comparison of two diabetic foot ulcer classification systems: the wagner and the University of Texas wound classification systems. Diabetes Care 2001;24:84-8.

54 Katsamouris AN, Giannoukas AD, Tsetis D, et al. Can ultrasound replace arteriography in the management of chronic arterial occlusive disease of the lower limb? Eur J Vasc Endovasc Surg 2001;21:155-9.

55 Lewis JEA, Owens DR. The pulse volume recorder as a measure of peripheral vascular status in people with diabetes mellitus. Diabetes Technol Ther 2010;12:75-80.

56 McPharlin M. Lower-Extremity arterial air plethysmography evaluation. Journal for Vascular Ultrasound 2012;36:135-42.

57 Lewis JE, Williams P, Davies JH. Non-Invasive assessment of peripheral arterial disease: automated ankle brachial index measurement and pulse volume analysis compared to duplex scan. SAGE Open Med 2016;4.

58 Khan TH, Farooqui FA, Niazi K. Critical review of the ankle brachial index. Curr Cardiol Rev 2008;4:101-6.

59 Daousi C, MacFarlane IA, Woodward A, et al. Chronic painful peripheral neuropathy in an urban community: a controlled comparison of people with and without diabetes. Diabet Med 2004;21:976-82

60 Meijer JWG, Smit AJ, Sonderen EV, et al. Symptom scoring systems to diagnose distal polyneuropathy in diabetes: the diabetic neuropathy symptom score. Diabet Med 2002;19:962-5.

61 Yang Z, Chen R, Zhang Y, et al. Scoring systems to screen for diabetic peripheral neuropathy. Cochrane Database Syst Rev 2014;22.

62 Mythili A, Kumar KD, Subrahmanyam KAV, et al. A comparative study of examination scores and quantitative sensory testing in diagnosis of diabetic polyneuropathy. Int J Diabetes Dev Ctries 2010;30:43-8.

63 WayneandPA. Clinical and laboratory Standards Institute. performance standards for antimicrobial susceptibility testing. In: 17th informational Suppl. Wayne, PA: CLSI, 2007: M100-17.

64 J A. JBP, Franklin R, CandLS. Institute, performance standards for antimicrobial susceptibility testing. Twenty-Fourth Informational Supplement 2014;32.

65 Huang Y, Cao Y, Zou M, et al. A comparison of tissue versus swab culturing of infected diabetic foot wounds. Int $J$ Endocrinol 2016;2016:1-6.

66 Logan BR, Zhang M-J, Klein JP. Regression models for hazard rates versus cumulative incidence probabilities in hematopoietic cell transplantation data. Biol Blood Marrow Transplant 2006;12:107-12.

67 Gray RJ. A Class of \$K\$-Sample Tests for Comparing the Cumulative Incidence of a Competing Risk. Ann Statist 1988;16:1141-54. 